\title{
Prognostic implication of PTPRH hypomethylation in non-small cell lung cancer
}

\author{
TAKASHI SATO ${ }^{1,2}$, KENZO SOEJIMA $^{1}$, ERI ARAI $^{2}$, JUNKO HAMAMOTO $^{1}$, HIROYUKI YASUDA $^{1}$, \\ DAISUKE ARAI $^{1}$, KOTA ISHIOKA ${ }^{1}$, KEIKO OHGINO $^{1}$, KATSUHIKO NAOKI $^{1}$, TAKASHI KOHNO ${ }^{3}$, \\ KOJI TSUTA ${ }^{4}$, SHUN-ICHI WATANABE ${ }^{5}$, YAE KANAI ${ }^{2}$ and TOMOKO BETSUYAKU ${ }^{1}$
}

${ }^{1}$ Division of Pulmonary Medicine, Department of Internal Medicine, Keio University School of Medicine, Tokyo 160-8582; Divisions of ${ }^{2}$ Molecular Pathology and ${ }^{3}$ Genome Biology, National Cancer Center Research Institute,

Departments of ${ }^{4}$ Pathology and Clinical Laboratories, Pathology Division and ${ }^{5}$ Thoracic Oncology, Thoracic Surgery Division, National Cancer Center Hospital, Tokyo 104-0045, Japan

Received April 16, 2015; Accepted May 11, 2015

DOI: 10.3892/or.2015.4082

\begin{abstract}
PTPRH is a receptor-type protein tyrosine phosphatase thought to be a potential regulator of tumorigenesis. The aim of the present study was to clarify the significance of $P T P R H$ expression and its regulation by DNA methylation in non-small cell lung cancer (NSCLC), especially in lung adenocarcinoma (LADC). PTPRH mRNA expression was examined in 89 NSCLC and corresponding non-cancerous tissues. The correlation between DNA methylation and PTPRH gene expression was investigated in another cohort that consisted of 145 patients with LADC, a major NSCLC subtype. Gene regulation by DNA methylation was assessed using a DNA methylation inhibitor. PTPRH mRNA expression was significantly upregulated in NSCLC. PTPRH DNA methylation was reduced in LADC samples and inversely correlated with mRNA expression. 5-Aza-2'-deoxycytidine treatment of lung cancer cell lines with low $P T P R H$ expression, restored mRNA PTPRH expression levels. Furthermore, low PTPRH methylation was associated with shorter recurrence-free survival
\end{abstract}

Correspondence to: Dr Kenzo Soejima, Division of Pulmonary Medicine, Department of Internal Medicine, Keio University School of Medicine, 35 Shinanomachi, Shinjuku-ku, Tokyo 160-8582, Japan E-mail: ksoejima@cpnet.med.keio.ac.jp

Abbreviations: NSCLC, non-small cell lung cancer; PTK, protein tyrosine kinase; EGFR, epidermal growth factor receptor; PTP, protein tyrosine phosphatase; SAP-1, stomach cancer-associated protein tyrosine phosphatase-1; LADC, lung adenocarcinoma; qRT-PCR, quantitative real-time reverse transcription-poly-merase chain reaction; MALDI-TOF MS, matrix-assisted laser desorption/ionization time-offlight mass spectrometry; 5-aza-dC, 5-aza-2'-deoxycytidine; ROC, receiver operating characteristic; TNM, tumor-node-metastasis; UICC, Union Internationale Contre le Cancer

Key words: non-small cell lung cancer, PTPRH, DNA methylation, hypomethylation, prognostic factor
$\left(\mathrm{P}=1.64 \times 10^{-4}\right)$ and overall survival $\left(\mathrm{P}=5.54 \times 10^{-5}\right)$. Multivariate analysis revealed that $P T P R H$ DNA methylation was an independent prognostic factor $\left(\mathrm{P}=6.88 \times 10^{-3}\right)$. It was confirmed that PTPRH is overexpressed in NSCLC. Furthermore, we determined that $P T P R H$ is epigenetically regulated by DNA hypomethylation, with prognostic implications for LADC.

\section{Introduction}

Lung cancer is the leading cause of cancer-related mortality worldwide (1). Non-small cell lung cancer (NSCLC) accounts for $\sim 85 \%$ of all lung malignancies and adenocarcinoma is the most common histological subtype. Although recent progress in diagnosis and treatment including molecular-targeted therapy have provided a considerable survival benefit, $\sim 40 \%$ of NSCLC are diagnosed at an advanced stage, with an overall 5 -year survival of $\sim 15 \%$ and recurrence rates remain high (2). Therefore, further identification of key molecular alterations in NSCLC is required.

Tyrosine phosphorylation is an important signaling mechanism in the regulation of various biological processes. Activation of protein tyrosine kinase (PTK) is a common feature of cancer and many drugs targeting PTKs, such as epidermal growth factor receptor (EGFR)-tyrosine kinase inhibitors, have been introduced. Protein tyrosine phosphatases (PTPs) also regulate tyrosine phosphorylation and are involved in cancer. Recent evidence has shown the relevance of PTPs either as tumor suppressors or oncoproteins (3). Epigenetic and genetic alterations in genes coding PTPs may be associated with cancer phenotypes.

PTPRH, also known as stomach cancer-associated PTP-1 (SAP-1), was first identified as a transmembrane-type PTP abundant in a subset of pancreatic and colorectal cancer cell lines (4). This enzyme belongs to the R3 subtype receptor-type PTP, together with PTPRB (also known as VE-PTP), PTPRJ (also known as DEP-1) and PTPRO and localizes to the microvilli of the brush border in the gastrointestinal tract (5). Ablation of PTPRH inhibits tumorigenesis in mice heterozygous for an adenomatous polyposis coli mutation $\left(A p c^{\mathrm{min} /+}\right)(6)$. Although it has been suggested that PTPRH regulates intestinal 
tumorigenesis, the mechanism is unclear. In contrast, PTPRH was found to be downregulated in advanced human hepatocellular carcinoma (7) and inhibited the proliferation of cultured cells $(8,9)$. Thus, the role of PTPRH is largely unknown and needs to be clarified in diseases including lung cancer.

Epigenetic changes such as aberrant DNA methylation in human cancers have been described (10). DNA hypermethylation in the 5'-UTR CpG-rich regions can block the expression of tumor suppressor genes. In NSCLC, silencing of specific genes such as RASSF1A, CDKN2A, RAR $\beta, M G M T$, $A P C, D A P K, F H I T$, and $C D H 13$ due to DNA hypermethylation around their promoter regions has been frequently observed (11). However, the role of DNA hypomethylation in permitting overexpression of specific genes is relatively poorly understood in NSCLC.

In the present study, we investigated the expression status of PTPRH in NSCLC. Next, it was elucidated that overexpression of PTPRH was caused by an underlying mechanism involving DNA hypomethylation. Then, the regulatory mechanism was confirmed in vitro using a DNA methylation inhibitor. Furthermore, it was determined that PTPRH DNA methylation was an independent prognostic factor for NSCLC patients.

\section{Materials and methods}

Patients and tissue samples. Two independent cohorts of lung cancer patients were investigated. The first cohort (LC-C1) comprised 89 paired samples of tumorous lung and corresponding non-cancerous tissues from patients with primary NSCLC who underwent lung resection at the Department of Thoracic Surgery, Keio University Hospital, Japan, between 2001 and 2006. Approval for institutional review of these samples was obtained in accordance with the requirements of the Keio University Institutional Review Board (IRB \#16-90-1). The second cohort (LC-C2) consisted of 145 paired samples of tumorous lung and corresponding non-cancerous tissues from patients with primary lung adenocarcinoma (LADC) who underwent lung resection at the National Cancer Center Hospital, Japan, between 1997 and 2008. These tissue specimens were provided by the National Cancer Center Biobank, Japan and the present study was also approved by the Ethics Committee of the National Cancer Center, Japan. All patients in the present study provided written informed consent. Clinicopathological parameters in both cohorts are summarized in Table I.

Cell lines. Three human lung cancer cell lines were used: A549 (adenocarcinoma), VMRC-LCD (adenocarcinoma) and EBC-1 (squamous cell carcinoma). A549 cells were purchased from the American Type Culture Collection (ATCC; Manassas, VA, USA). VMRC-LCD and EBC-1 cells were purchased from the Health Science Research Resources Bank (Osaka, Japan). All cell lines were cultured according to the supplier's instructions.

cDNA microarray analysis. GeneChip Human Genome 2.0 array (Affymetrix, Inc., Santa Clara, CA, USA) was used to monitor the expression profiles of the LC-C1 samples. Total RNA was extracted from tumorous tissues and paired non-cancerous lung tissues using TRIzol (Life Technologies,
Carlsbad, CA, USA). The labeled cRNA was prepared using standard Affymetrix protocols. The signal intensities of the probe sets were normalized using the Affymetrix Power Tools RMA method implemented using Resolver software (Rosetta Biosoftware, Seattle, WA, USA).

Quantitative real-time reverse transcription-polymerase chain reaction ( $q R T-P C R)$ analysis. Total RNA extracted from 62 tumorous and 17 non-cancerous tissues in $\mathrm{LC}-\mathrm{C} 1$, 111 tumorous and 96 non-cancerous tissues in LC-C2 and lung cancer cell lines, was reverse-transcribed to cDNA using TaqMan reverse transcription reagents or SuperScript III reverse transcriptase (both from Life Technologies). For qRT-PCR analysis, TaqMan gene expression assays were used for human PTPRH (Hs00936195_m1, \#4331182) and human glyceraldehyde-3-phosphate dehydrogenase (GAPDH; \#4310884E, both from Life Technologies) to normalize input cDNA. Quantitative analysis was performed using Applied Biosystems 7500 Fast-Real Time PCR system (Life Technologies). All assays were performed in triplicate.

Infinium assay. Genomic DNA was extracted from all LC-C2 tissue samples and lung cancer cell lines using a QIAamp DNA mini kit (Qiagen, Valencia, CA, USA). Bisulfite conversion using an EZ DNA Methylation-Gold kit (Zymo Research, Irvine, CA, USA) was carried out on $500 \mathrm{ng}$ aliquots of DNA. Subsequently, DNA methylation status at $27,578 \mathrm{CpG}$ loci was examined at single-CpG resolution using the Infinium HumanMethylation27 Bead array (Illumina, San Diego, CA, USA). An Evo robot (Tecan, Männedorf, Switzerland) was used for automated sample processing. After whole genome amplification and hybridization, the specifically hybridized DNA was fluorescence-labeled by a single-base extension reaction and detected using a BeadScan reader (Illumina) in accordance with the manufacturer's protocols. The data were then assembled using GenomeStudio methylation software (Illumina). At each $\mathrm{CpG}$ site, the ratio of the fluorescence signal was measured using a methylated probe relative to the sum of the methylated and unmethylated probes, such as the $\beta$-value, which ranges from 0 to 1 and reflects the methylation level of an individual CpG site. The reliability of DNA methylation levels ( $\beta$-values) determined by the Infinium assay was verified in our previous studies (12-14).

DNA methylation analysis with the MassARRAY system. Bisulfite treatment using an EpiTect Bisulfite Kit (Qiagen $\mathrm{GmbH}$, Hilden, Germany) was carried out on $250 \mathrm{ng}$ of genomic DNA extracted from LC-C2 samples, in accordance with the manufacturer's protocol. DNA methylation levels containing the CpG site $\operatorname{cg} 11261264$ in the Infinium assay were evaluated quantitatively using the MassARRAY platform (Sequenom, San Diego, CA, USA). This method utilizes base-specific cleavage and matrix-assisted laser desorption/ionization time-of-flight mass spectrometry (MALDI-TOF MS) (15). Specific PCR primers for bisulfite-converted DNA were designed using the EpiDesigner software package (www. epidesigner.com; Sequenom). The forward and reverse primers used were 5'-TTGTGTGTTGTTTGAAGTTAGTGTTT-3' and 5'-CTAАACCTAAААCTCCTAАATCCCC-3', respectively. A T7-promoter tag (5'-CAGTAATACGACTCACTATAG 
Table I. Clinicopathological parameters of patients with NSCLCs in LC-C1 and LC-C2.

\begin{tabular}{llr}
\hline $\begin{array}{l}\text { Clinicopathological } \\
\text { parameters }\end{array}$ & $\begin{array}{l}\text { LC-C1 } \\
(\mathrm{n}=89)\end{array}$ & $\begin{array}{r}\text { LC-C2 } \\
(\mathrm{n}=145)\end{array}$ \\
\hline
\end{tabular}

Age (years)

Median

68

Interquartile range

60-75

Gender

Male

Female

Smoking status

(pack-year)

Median

Interquartile range

$0-60$

Histological type

Adenocarcinoma

Squamous

cell carcinoma

Large

cell carcinoma

Others

6

Tumor size $(\mathrm{cm})$

Median

Interquartile range

$2.5-4.0$

Tumor stage
T1

T2

T3-4

41

30

18

Nodal status

No

N1

N2-3

60

14

15

Metastatic status

M0

M1

Pathological TNM stage

I

II

III-IV

EGFR mutation

Wild-type

K-ras mutation

Wild-type

84

Mutant

5 forward primer to balance the PCR. To overcome PCR bias in DNA methylation analysis, we optimized the annealing temperature and type of DNA polymerase: 0, 50 and $100 \%$ methylated control DNA (EpiTect methylated human control DNA; Qiagen) was used as template to test the linearity of the protocol. HotStar Taq DNA polymerase (Qiagen) was used for the PCR. The PCR products were used as a template for in vitro transcription and $\mathrm{RNase} A$-mediated cleavage reaction using an EpiTYPER reagent kit (Sequenom). The fragmented samples were dispensed onto a SpectroCHIP array and then detected on a MassARRAY analyzer compact MALDI-TOF MS instrument. The data were visualized using EpiTYPER Analyzer software v1.0 (Sequenom). The DNA methylation level (\%) at each $\mathrm{CpG}$ site was determined by comparing the signal intensities of methylated and non-methylated templates. Experiments were performed in triplicate for each sample-CpG site and the mean value for the three experiments was used as the DNA methylation level.

5-Aza-2'-deoxycytidine (5-aza-dC) treatment. A549, VMRC-LCD and EBC-1 cells were seeded at a density of $9 \times 10^{5}$ cells $/ 15 \mathrm{~cm}$ dish on day 0 and then allowed to attach for a 24-h period. Then, 5-aza-dC (Sigma-Aldrich, St. Louis, MO, USA) was added to a final concentration of $5 \mu \mathrm{M}$. Cells were passaged at a subculture ratio of 1:2 on day 3. 5-Aza-dC was added again to the same final concentration $24 \mathrm{~h}$ after replating. Since toxicity was observed during preliminary experiments, the final concentration of 5-aza-dC was reduced to $0.5 \mu \mathrm{M}$ for EBC- 1 cells. Genomic DNA and total RNA were extracted from all cells on days 3 and 6 .

Statistical analysis. Differences in mRNA expression or DNA methylation levels between cancerous and non-cancerous tissues were investigated using the Wilcoxon signed-rank or Mann-Whitney U test, respectively. Correlation between PTPRH mRNA expression levels obtained by microarray and qRT-PCR, as well as correlation between DNA methylation and mRNA expression levels of PTPRH, were examined using Spearman's correlation test. DNA methylation levels obtained by the MassARRAY system were compared with those by the Infinium assay using the Pearson's correlation test. The correlation between mRNA expression or DNA methylation levels of PTPRH and clinicopathological factors was examined using Spearman's correlation, Mann-Whitney U and Kruskal-Wallis test. Survival curves for patients with LADC were analyzed by the Kaplan-Meier method and log-rank test. The PTPRH DNA methylation cutoff level was determined in order to maximize the sensitivity and specificity for recurrence by receiver operating characteristic (ROC) curve analysis (16). Multivariate analysis of the influence of variables on recurrence-free survival was performed using the Cox proportional hazards model. Statistical analyses were performed using SPSS 20.0 (SPSS, IBM, Chicago, IL, USA) and GraphPad Prism 5.0 software (GraphPad Software, La Jolla, CA, USA). All P-values were two-sided and $\mathrm{P}<0.05$ was considered statistically significant.

\section{Results}

GGAGAAGGCT-3') was added to the reverse primer for PTPRH mRNA expression is increased in NSCLC. PTPRH gene expression in LC-C1 was investigated by cDNA micro- 
A

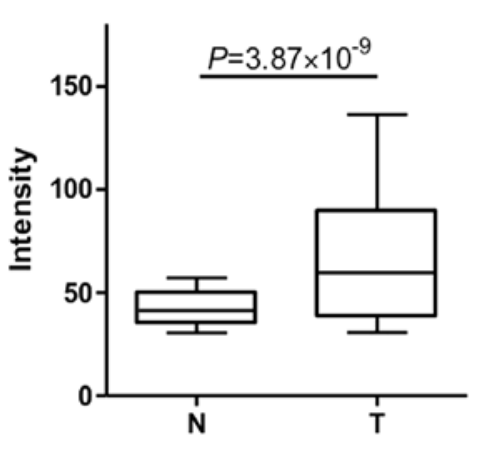

B

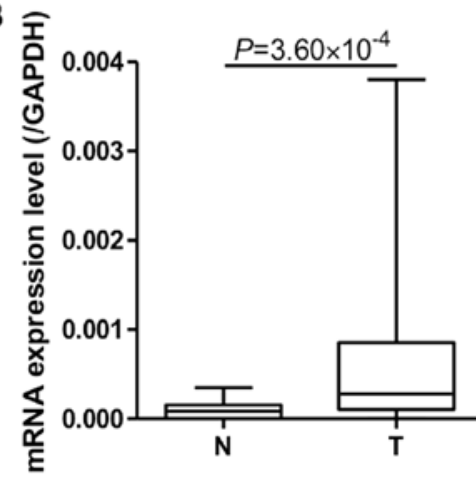

C

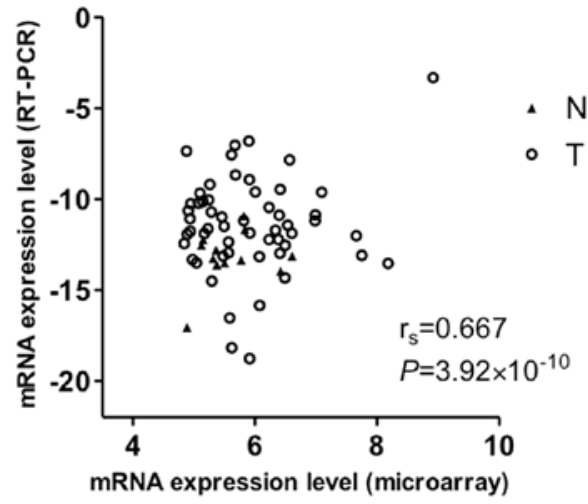

Figure 1. PTPRH is significantly upregulated in NSCLC. (A) Microarray gene expression for PTPRH in samples of N and of T in LC-C1. PTPRH is significantly upregulated in T samples. (B) mRNA expression levels of PTPRH in N and the corresponding T samples in LC-C1 obtained by qRT-PCR. PTPRH expression is also significantly higher in T samples. (C) Correlation of PTPRH mRNA expression between microarray (X-axis; normalized intensity on a $\log _{2}$ scale) and qRT-PCR (Y-axis; relative PTPRH/GAPDH expression on a $\log _{2}$ scale) analysis for LC-C1 samples. The variables are well correlated. NSCLC, non-small cell lung cancer; $\mathrm{N}$, non-cancerous lung tissue; T, corresponding tumorous tissue; qRT-PCR, quantitative real-time reverse transcription-polymerase chain reaction.

A

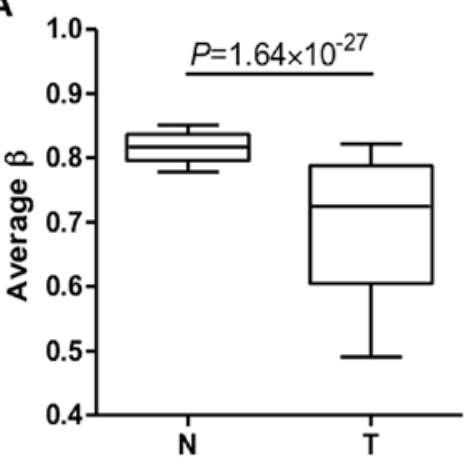

B

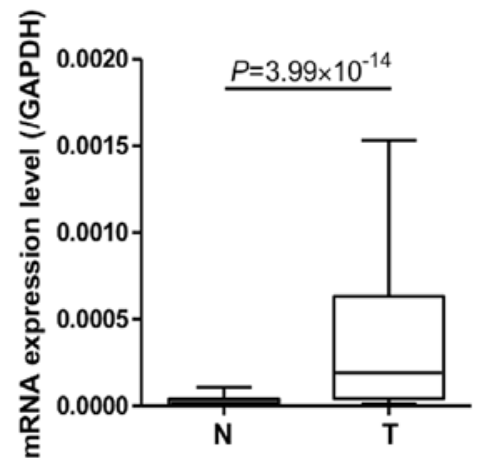

C

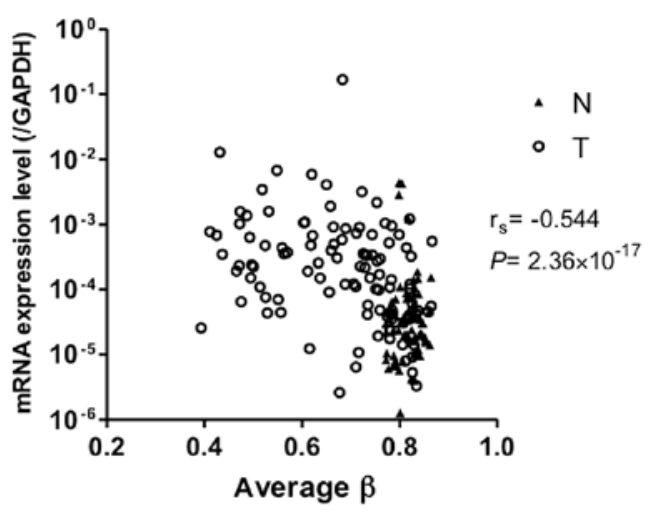

Figure 2. Correlation between DNA methylation and mRNA expression levels for PTPRH. DNA methylation (average values) (A) and mRNA expression levels (B) for PTPRH in samples of N and of T in LC-C2 were determined by the Infinium assay and qRT-PCR analysis, respectively. DNA methylation levels for PTPRH were significantly lower in $\mathrm{T}$ than in $\mathrm{N}$ samples and levels of PTPRH mRNA expression were significantly higher in T than in N samples. (C) Correlation of DNA methylation (average values) and mRNA expression levels for PTPRH in LC-C2 samples. PTPRH mRNA expression levels were inversely correlated with DNA methylation of the single CpG site. These results suggested that PTPRH DNA hypomethylation may result in increased mRNA expression in tissue samples from the same cohort. N, non-cancerous lung tissue; T, corresponding tumorous tissue; qRT-PCR, quantitative real-time reverse transcription-polymerase chain reaction.

array. The PTPRH mRNA expression levels were significantly higher in tumorous tissues compared with the corresponding non-cancerous tissues (Fig. 1A). We performed qRT-PCR on PTPRH to confirm the microarray data for 62 cancerous and 17 non-cancerous tissues whose samples were still available. Again, the PTPRH mRNA expression levels were significantly higher in tumorous tissues compared with non-cancerous tissues (Fig. 1B). The PTPRH mRNA expression data correlated well with that determined by qRT-PCR and microarray analysis $\left(\mathrm{r}_{\mathrm{s}}=0.667 ; \mathrm{P}=3.92 \times 10^{-10}\right.$; Fig. $\left.1 \mathrm{C}\right)$.

The correlation between PTPRH gene expression and clinicopathological parameters are summarized in Table II. Tumor-node-metastasis (TNM) classification was performed according to the Union Internationale Contre le Cancer (UICC)-6 staging system for NSCLC. Distribution of age, gender, histological type, pathological TNM stage, EGFR and $K$-ras mutation status except for smoking status did not significantly correlate with PTPRH mRNA expression.
PTPRH DNA methylation is reduced and inversely correlated with $m R N A$ expression in $L A D C$. Based on the hypothesis that altered mRNA expression may be caused by aberrant DNA methylation, we investigated the DNA methylation status of PTPRH in LC-C2 samples for which genome-wide DNA methylation profiles were available. As there was no significant correlation between PTPRH mRNA expression levels and histological type, we used LC-C2 samples and focused on LADC, a major histological type in LC-C1, for further analysis of the epigenetic regulation of PTPRH. DNA methylation levels of the CpG site cg11261264, located in PTPRH intron 1, were significantly decreased in tumorous compared with non-cancerous tissues (Fig. 2A). We also examined PTPRH mRNA expression levels in LC-C2 samples by qRT-PCR. This showed that PTPRH mRNA expression levels were again higher in tumorous tissues compared with the corresponding non-cancerous (Fig. 2B) and inversely correlated with DNA methylation of this single $\mathrm{CpG}$ site (Fig. 2C). These data 
Table II. Correlation between mRNA expression levels (microarray) of PTPRH and clinicopathological parameters of patients with non-small cell lung cancers.

\begin{tabular}{|c|c|c|c|}
\hline $\begin{array}{l}\text { Clinicopatho- } \\
\text { logical } \\
\text { parameters }\end{array}$ & No. & $\begin{array}{l}\text { Median intensity } \\
\text { (interquartile } \\
\text { range) }\end{array}$ & $\mathrm{P}^{\mathrm{a}}$-value \\
\hline Age (years) & & & $\begin{array}{l}5.94 \times 10^{-1 b} \\
\left(r_{s}=-0.057\right)\end{array}$ \\
\hline \multicolumn{4}{|l|}{ Gender } \\
\hline Male & 56 & $63.9(41.4-95.1)$ & $9.56 \times 10^{-2 \mathrm{c}}$ \\
\hline Female & 33 & $47.3(37.7-84.2)$ & \\
\hline \multicolumn{4}{|l|}{ Smoking status } \\
\hline (pack-year) & & & $\begin{array}{l}6.73 \times 10^{-3 b} \\
\left(r_{s}=0.287\right)\end{array}$ \\
\hline \multicolumn{4}{|l|}{ Histological type } \\
\hline $\begin{array}{l}\text { Adenocarcinoma } \\
\text { Squamous cell }\end{array}$ & 54 & $49.9(35.4-84.9)$ & $1.51 \times 10^{-1 \mathrm{~d}}$ \\
\hline $\begin{array}{l}\text { carcinoma } \\
\text { Large cell }\end{array}$ & 24 & $62.0(46.1-91.2)$ & \\
\hline carcinoma & 6 & $84.9(59.6-114.3)$ & \\
\hline Others & 5 & $74.9(64.0-97.3)$ & \\
\hline \multicolumn{4}{|l|}{ Tumor stage } \\
\hline $\mathrm{T} 1$ & 41 & $49.5(38.9-85.0)$ & $3.48 \times 10^{-1 b}$ \\
\hline $\mathrm{T} 2$ & 30 & $71.0(36.7-92.5)$ & \\
\hline T3-4 & 18 & $60.2(38.2-105.7)$ & \\
\hline \multicolumn{4}{|l|}{ Nodal status } \\
\hline NO & 60 & $60.2(41.5-93.6)$ & $2.81 \times 10^{-1 b}$ \\
\hline N1 & 14 & $60.5(34.7-89.6)$ & \\
\hline $\mathrm{N} 2-3$ & 15 & $47.3(35.3-87.5)$ & \\
\hline \multicolumn{4}{|l|}{ Metastatic status } \\
\hline M0 & 87 & $56.8(37.7-87.5)$ & $7.63 \times 10^{-2 b}$ \\
\hline M1 & 2 & $119.4(114.3-124.5)$ & \\
\hline \multicolumn{4}{|l|}{ Pathological } \\
\hline \multicolumn{4}{|l|}{ TNM stage } \\
\hline $\mathrm{I}$ & 50 & $57.8(38.9-92.5)$ & $8.72 \times 10^{-1 b}$ \\
\hline II & 12 & $62.0(44.8-83.9)$ & \\
\hline III-IV & 27 & $59.9(35.3-114.3)$ & \\
\hline \multicolumn{4}{|l|}{$E G F R$ mutation } \\
\hline Wild-type & 63 & $63.7(43.8-92.5)$ & $1.56 \times 10^{-1 b}$ \\
\hline Mutant & 26 & $46.1(35.9-84.2)$ & \\
\hline \multicolumn{4}{|l|}{$K$-ras mutation } \\
\hline Wild type & 84 & $58.2(38.6-89.8)$ & $7.91 \times 10^{-1 b}$ \\
\hline Mutant & 5 & $59.9(30.6-84.9)$ & \\
\hline
\end{tabular}

${ }^{\text {aP }}$-values of $<0.05$ are bold print. ${ }^{\text {b}}$ Spearman's correlation test. 'MannWhitney U-test. ${ }^{\mathrm{d} K r u s k a l-W a l l i s ~ t e s t ; ~ T N M, ~ t u m o r-n o d e-m e t a s t a s i s . ~}$

suggested that PTPRH DNA hypomethylation may result in increased mRNA expression in tissue samples from the same cohort.
Table III. CpG sites analyzed by the MassARRAY system.

\begin{tabular}{ll}
\hline CpG site & \multicolumn{1}{c}{ Position $^{\mathrm{a}}$} \\
\hline CpG_1 & Chromosome 19: 55,720,456 \\
CpG_2 (not covered) & Chromosome 19: 55,720,467 \\
CpG_3 & Chromosome 19: 55,720,485 \\
CpG_4 (not covered) & Chromosome 19: 55,720,516 \\
CpG_5 & Chromosome 19: 55,720,534 \\
CpG_6 & Chromosome 19: 55,720,554 \\
CpG_7 (not covered) & Chromosome 19: 55,720,565 \\
CpG_8 (not covered) & Chromosome 19: 55,720,668 \\
CpG_9.10 & Chromosome 19: 55,720,728. 55,720,737 \\
CpG_11 & Chromosome 19: 55,720,777 \\
CpG_12 & Chromosome 19: 55,720,786 \\
CpG_13 & Chromosome 19: 55,720,817
\end{tabular}

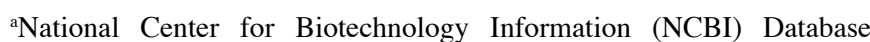
(Genome Build 37). ${ }^{\mathrm{b}} \mathrm{CpG}$ site identical to $\operatorname{cg} 11261264$ in the Infinium assay.

PTPRH gene expression is regulated by DNA hypomethylation. To improve understanding of the influence of DNA methylation on PTPRH gene expression, lung cancer cell lines were treated with the DNA methylation inhibitor, 5-aza-dC. In three lung cancer cell lines A549, VMRC-LCD, and EBC-1 with low PTPRH mRNA expression, 5-aza-dC treatment induced a marked reduction of DNA methylation and restored PTPRH mRNA expression levels (Fig. 3). These data suggested that increased expression of PTPRH was primarily regulated by DNA hypomethylation in LADC.

DNA hypomethylation of PTPRH as a prognostic factor. Because investigation of DNA methylation by the MassARRAY system allowed a comprehensive coverage of $\mathrm{CpG}$ sites, we assessed DNA methylation levels at the relatively $\mathrm{CpG}$-rich region containing the $\mathrm{CpG}$ site cg11261264 in the same LC-C2 samples using the MassARRAY system (Genomic positions of the CpG sites are shown in Table III). DNA methylation levels obtained by the MassARRAY system and Infinium assay correlated well $\left(\mathrm{r}=0.952, \mathrm{P}=1.44 \times 10^{-73}\right)$, confirming the reliability of the latter assay. DNA methylation patterns quantified at each analyzed $\mathrm{CpG}$ site in non-cancerous, tumorous tissue with no recurrence and those with recurrence are shown

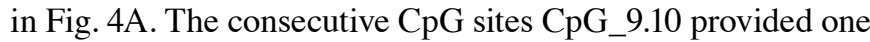
measured value as a 'CpG unit' by the MassARRAY system. DNA methylation levels at most CpG sites showed a significant decrease in tumorous tissues with recurrence compared to those with no recurrence. The correlation between the DNA methylation levels of cancerous tissues at CpG_9.10 and clinicopathological parameters are summarized in Table IV. DNA hypomethylation at this $\mathrm{CpG}$ unit was significantly correlated with male gender, heavy smoking status, advanced pathological stage and wild-type EGFR.

Kaplan-Meier analysis based on the optimal cutoff determined by ROC curve analysis showed that patients with hypomethylation at the $\mathrm{CpG}$ unit had a shorter recurrence-free and overall survival compared with patients with hypermeth- 
A

A549
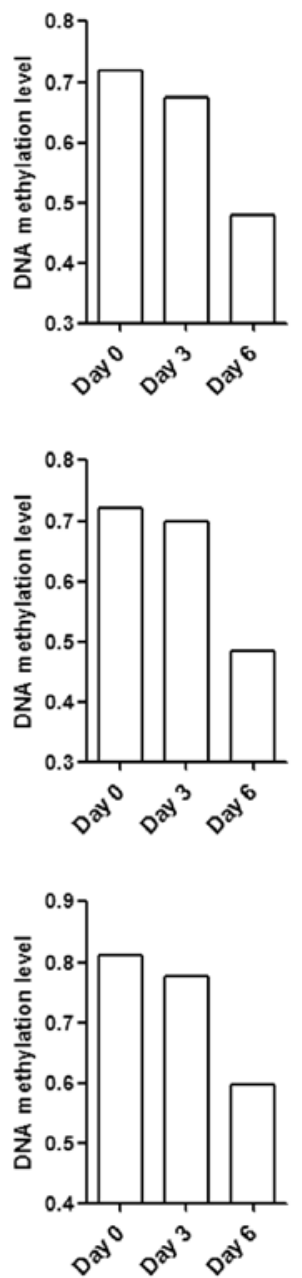

B
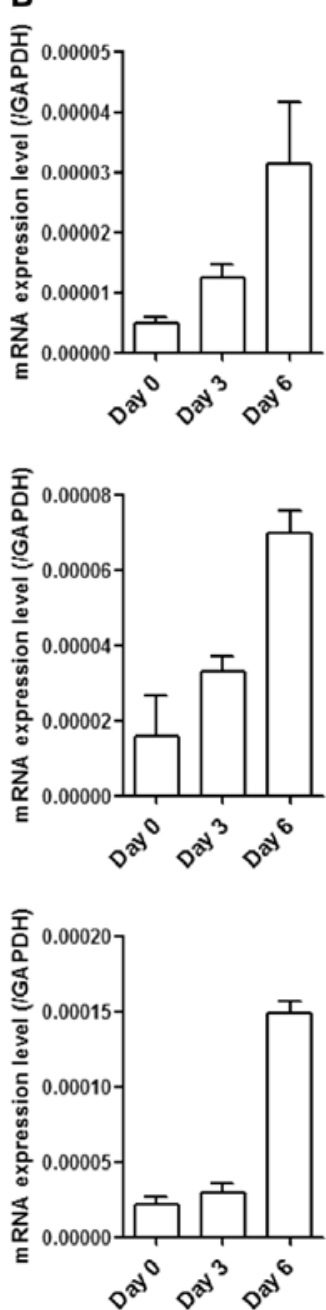

Figure 3. DNA methylation and mRNA expression levels after 5-aza2'-deoxycytidine (5-aza-dC) treatment. (A) DNA methylation (average values) and (B) mRNA expression levels for PTPRH were determined by the Infinium assay and qRT-PCR analysis, respectively. The error bars represent the standard deviation for triplicate qRT-PCR analyses. DNA methylation and mRNA expression levels on days 3 and 6 were compared with those of untreated cells. After 5-aza-dC treatment, reduction of DNA methylation levels and restoration of the PTPRH mRNA expression levels were observed in both of the cell lines used. qRT-PCR, quantitative real-time reverse transcription-polymerase chain reaction.

ylation (Fig. 4B; $\mathrm{P}=1.64 \times 10^{-4}$ and $\mathrm{P}=5.54 \times 10^{-5}$, respectively). Since multiple covariates can affect patient survival, we performed multivariate analysis to confirm that the DNA methylation status of $P T P R H$ is an independent prognostic factor for LADC patients. In multivariate Cox proportional hazards regression analysis, PTPRH DNA methylation, together with gender and pathological TNM status, emerged as an independent prognostic factor for recurrence-free survival (Table V).

\section{Discussion}

Although some studies have demonstrated the involvement of PTPRH in cancer, especially in intestinal tumorigenesis $(4,12,17)$, its role in lung cancer and the molecular mechanisms underlying its regulation have not been clarified. In the present study, we examined PTPRH expression
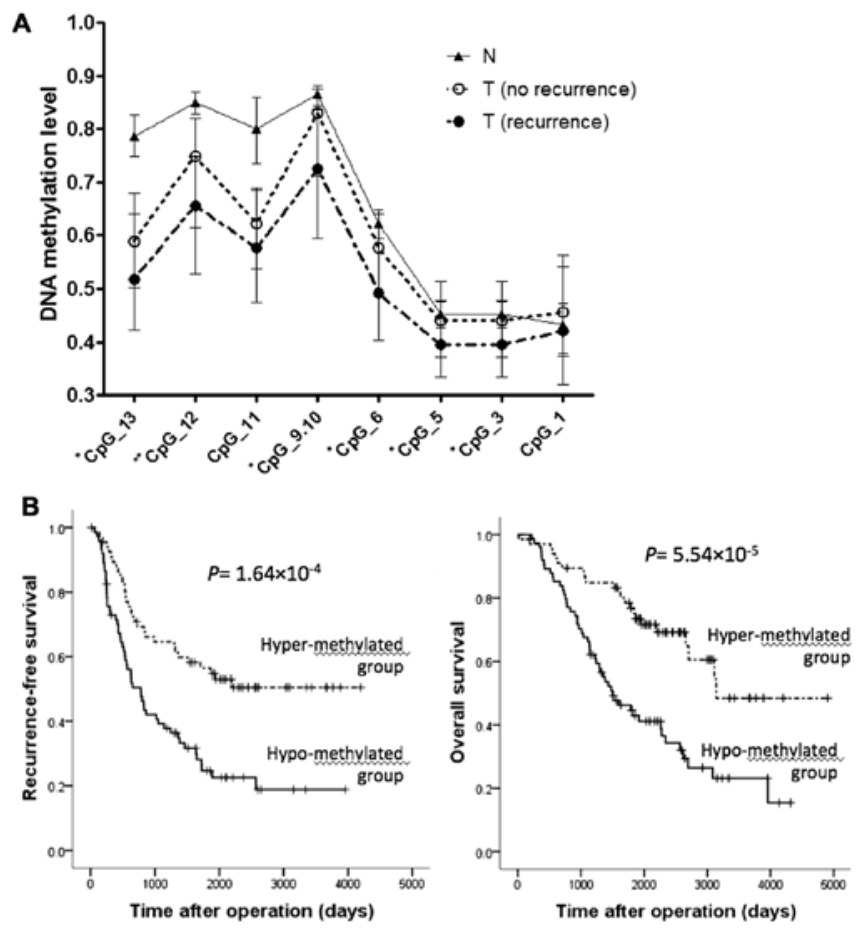

Figure 4. (A) Median DNA methylation levels of PTPRH in $\mathrm{N}$ and $\mathrm{T}$ with no recurrence and those with recurrence in LC-C2. DNA methylation levels of each $\mathrm{CpG}$ unit were evaluated quantitatively using the MassARRAY system. The error bars are defined by $25 / 75 \%$ quartiles. DNA methylation levels at most $\mathrm{CpG}$ sites showed a significant decrease in tumorous tissue with recurrence compared with those with no recurrence. Significantly different methylated $\mathrm{CpG}$ sites are marked as ${ }^{*} \mathrm{P}<0.05$ and ${ }^{* *} \mathrm{P}<0.01$ ). (B) KaplanMeier survival curves of patients with $P T P R H$ hypermethylation $(>0.782$ at CpG_9.10) and hypomethylation $(\leq 0.782)$. The cutoff was determined by ROC curve analysis. The recurrence-free $\left(\mathrm{P}=1.64 \times 10^{-4}\right)$ and overall $\left(\mathrm{P}=5.54 \times 10^{-5}\right)$ survival rates of patients with hypomethylation were significantly lower compared to those of patients with hypermethylation (log-rank test). N, non-cancerous lung tissue; $\mathrm{T}$, corresponding tumorous tissue; ROC, receiver operating characteristic.

in NSCLC and focused on its regulation by DNA methylation and its clinicopathological implications.

First, we showed that $P T P R H$ expression is increased in NSCLC using cDNA microarray analysis. mRNA expression levels obtained from qRT-PCR confirmed the microarray data. While PTPRH expression was found to be increased in human colon and pancreatic cancer $(4,17)$ and reduced in advanced human hepatocellular carcinoma (7), to our knowledge no previous study has examined its expression in lung cancer. Our data from LC-C1 samples suggested that PTPRH may have a significant role in NSCLC and be associated with its clinicopathological features. In fact, $P T P R H$ expression was associated with smoking status that is a well-known risk factor for NSCLC. However, no other association was observed at a statistically significant level.

As PTPRH was upregulated in NSCLC, we became interested in the molecular mechanisms underlying its increased expression. Recent studies analyzed the genome-wide DNA methylation profiles of $\operatorname{LADC}(13,14)$. We investigated the DNA methylation levels of LC-C2 samples using data from the Infinium assay. As LC-C 2 consists of patients with LADC only, we focused on PTPRH in LADC, a major subtype of 
Table IV. Correlation between DNA methylation levels of PTPRH and clinicopathological parameters of patients with lung adenocarcinomas.

\begin{tabular}{|c|c|c|c|}
\hline Clinicopathological parameters & No. & $\begin{array}{l}\text { Median DNA methylation } \\
\text { level (interquartile range) }\end{array}$ & P-value ${ }^{a}$ \\
\hline Years of age & & & $\begin{array}{l}7.01 \times 10^{-2 b} \\
\left(r_{s}=0.153\right)\end{array}$ \\
\hline \multicolumn{4}{|l|}{ Gender } \\
\hline Male & 81 & $0.723(0.597-0.830)$ & \multirow[t]{2}{*}{$3.05 \times 10^{-3 c}$} \\
\hline Female & 64 & $0.833(0.690-0.870)$ & \\
\hline Smoking status (pack-year) & & & $\begin{array}{l}\mathbf{3 . 5 1 \times 1 0} \mathbf{1 0}^{-3 \mathrm{~b}} \\
\left(\mathrm{r}_{\mathrm{s}}=-0.244\right)\end{array}$ \\
\hline \multicolumn{4}{|l|}{ Tumor stage } \\
\hline $\mathrm{T} 1$ & 64 & $0.793(0.672-0.850)$ & \multirow[t]{3}{*}{$3.15 \times 10^{-1 b}$} \\
\hline $\mathrm{T} 2$ & 63 & $0.727(0.593-0.850)$ & \\
\hline T3-4 & 18 & $.0 .737(0.627-0.872)$ & \\
\hline \multicolumn{4}{|l|}{ Nodal status } \\
\hline No & 94 & $0.793(0.627-0.860)$ & \multirow[t]{3}{*}{$1.96 \times 10^{-1 b}$} \\
\hline $\mathrm{N} 1$ & 24 & $0.768(0.602-0.0 .845)$ & \\
\hline $\mathrm{N} 2-3$ & 27 & $0.713(0.643-0.797)$ & \\
\hline \multicolumn{4}{|l|}{ Pathological TNM stage } \\
\hline I & 83 & $0.807(0.627-0.867)$ & \multirow[t]{3}{*}{$1.57 \times 10^{-2 b}$} \\
\hline II & 31 & $0.767(0.653-0.853)$ & \\
\hline III & 31 & $0.703(0.600-0.790)$ & \\
\hline \multicolumn{4}{|l|}{ EGFR mutation } \\
\hline Wild-type & 73 & $0.717(0.547-0.843)$ & \multirow[t]{2}{*}{$7.28 \times 10^{-3 b}$} \\
\hline Mutant & 48 & $0.820(0.647-0.873)$ & \\
\hline \multicolumn{4}{|l|}{$K$-ras mutation } \\
\hline Wild-type & 109 & $0.767(0.617-0.857)$ & \multirow[t]{2}{*}{$9.39 \times 10^{-2 b}$} \\
\hline Mutant & 12 & $0.665(0.505-0.755)$ & \\
\hline
\end{tabular}

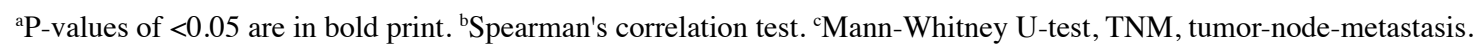

Table V. Multivariate analysis of predictive factors for recurrence-free survival in patients with LADCs (Cox proportional hazard model).

\begin{tabular}{|c|c|c|}
\hline \multirow{2}{*}{$\begin{array}{l}\text { Variables } \\
\text { Gender }\end{array}$} & \multicolumn{2}{|c|}{$\begin{array}{c}\text { Multivariate analysis } \\
\text { Hazard ratio } \\
\text { (95\% confidence interval) }\end{array}$} \\
\hline & $1.801(1.012-3.205)$ & $4.53 \times 10^{-2}$ \\
\hline Smoking status (pack-year) & $1.000(1.000-1.001)$ & $1.72 \times 10^{-1}$ \\
\hline Pathological TNM stage & $1.468(1.276-1.690)$ & $8.69 \times 10^{-8}$ \\
\hline$E G F R$ mutation & $1.490(0.923-2.407)$ & $1.03 \times 10^{-1}$ \\
\hline PTPRH DNA methylation & $0.134(0.031-0.576)$ & $6.88 \times 10^{-3}$ \\
\hline
\end{tabular}

Bold, P-values of $<0.05$. TNM, tumor-node-metastasis.

NSCLC, to concentrate on the correlation between measured values and clinicopathological parameters and patient survival. This showed that PTPRH DNA methylation was reduced in LADC and inversely correlated with mRNA expression. Several studies identified tumor-specific hypermethylation of other types of PTPs, such as PTPRO $(18,19)$, PTPRD (20), PTPRG (21,22), PTPN6 (23-25) and PTPN13 (26), reviewed by Jacob and Motiwala (27), and Julien et al (3). However, tumor-specific hypomethylation of PTP has not been reported. 5-aza-dC treatment of human lung cancer cell lines with low PTPRH expression restored PTPRH mRNA expression levels indicating that $P T P R H$ is reactivated by DNA hypomethylation during lung tumorigenesis.

We speculated that PTPRH may play an oncogenic role in lung tumorigenesis because PTPRH is thought to promote intestinal tumorigenesis (6). We attempted a transient knockdown of PTPRH using siRNA in lung cancer cell lines. However, we were unable to find any lung cancer cell lines where cell proliferation was inhibited by its knockdown (data not shown). Although PTPRH was shown to have a capacity to activate Src (28), Src kinase may not be responsible for its oncogenic properties. Sadakata et al reported that ablation of PTPRH did not reduce the levels of c-Src activity in $A p c^{\mathrm{min} /+}$ mice (6) 
and we did not observe changes in Src phosphorylation (Y416) in PTPRH-knockdown cells (data not shown). Recently, the substrate specificity of the R3 subtype of receptor-type PTPs toward receptor tyrosine kinases was described (29). Further studies are needed to clarify the mechanisms downstream of PTPRH.

We further confirmed the data from the Infinium assay by using the MassARRAY platform and investigated the clinicopathological and prognostic significance of $P T P R H$ DNA methylation in LADC. PTPRH DNA hypomethylation was significantly correlated with male gender, heavy smoking status, pathological stage and wild-type EGFR. Seo et al described the correlation between PTPRH expression and $K$-ras mutations in colorectal cancer (17). However, no correlation was found in the present study, probably due to differences in histology. Although the underlying significance of these results and the precise function of PTPRH were not clarified in the present study, our analysis explored PTPRH hypomethylation as a novel prognostic factor. To the best of our knowledge, this is the first study to show that PTPRH is regulated by DNA methylation and its hypomethylation is related to poor prognosis in LADC.

In conclusion, PTPRH is upregulated in NSCLC and it is regulated by DNA hypomethylation in LADC. Moreover, the DNA methylation status of PTPRH has been identified as a novel prognostic factor for LADC. Further studies are warranted to clarify its molecular role in the development of NSCLC.

\section{Acknowledgements}

We would like to thank Ms. Mikiko Shibuya for her excellent technical assistance. The present study was supported in part by the Grants-in-Aid for Scientific Research from the Japan Society for the Promotion of Science to T.S. (Grant no. 26870569) and K.S. (Grant no. 22590870) and the Program for Promotion of Fundamental Studies in Health Sciences (10-42) of the National Institute of Biomedical Innovation (NiBio), Japan. T.S. is an awardee of a research resident fellowship from the Foundation for Promotion of Cancer Research in Japan.

\section{References}

1. Siegel R, Naishadham D and Jemal A: Cancer statistics, 2013. CA Cancer J Clin 63: 11-30, 2013.

2. Liu L, Zhao E, Li C, Huang L, Xiao L, Cheng L, Huang X, Song Y and Xu D: TRIM28, a new molecular marker predicting metastasis and survival in early-stage non-small cell lung cancer. Cancer Epidemiol 37: 71-78, 2013.

3. Julien SG, Dubé N, Hardy S and Tremblay ML: Inside the human cancer tyrosine phosphatome. Nat Rev Cancer 11: 35-49, 2011.

4. Matozaki T, Suzuki T, Uchida T, Inazawa J, Ariyama T, Matsuda K, Horita K, Noguchi H, Mizuno H, Sakamoto C, et al: Molecular cloning of a human transmembrane-type protein tyrosine phosphatase and its expression in gastrointestinal cancers. J Biol Chem 269: 2075-2081, 1994.

5. Matozaki T, Murata Y, Mori M, Kotani T, Okazawa $\mathrm{H}$ and Ohnishi H: Expression, localization, and biological function of the R3 subtype of receptor-type protein tyrosine phosphatases in mammals. Cell Signal 22: 1811-1817, 2010.

6. Sadakata H, Okazawa H, Sato T, Supriatna Y, Ohnishi H, Kusakari S, Murata Y, Ito T, Nishiyama U, Minegishi T, et al: SAP-1 is a microvillus-specific protein tyrosine phosphatase that modulates intestinal tumorigenesis. Genes Cells 14: 295-308, 2009.
7. Nagano H, Noguchi T, Inagaki K, Yoon S, Matozaki T, Itoh $\mathrm{H}$, Kasuga M and Hayashi Y: Downregulation of stomach cancer-associated protein tyrosine phosphatase-1 (SAP-1) in advanced human hepatocellular carcinoma. Oncogene 22: 4656-4663, 2003.

8. Noguchi T, Tsuda M, Takeda H, Takada T, Inagaki K, Yamao T, Fukunaga K, Matozaki T and Kasuga M: Inhibition of cell growth and spreading by stomach cancer-associated protein-tyrosine phosphatase-1 (SAP-1) through dephosphorylation of p130cas. J Biol Chem 276: 15216-15224, 2001.

9. Takada T, Noguchi T, Inagaki K, Hosooka T, Fukunaga K, Yamao T, Ogawa W, Matozaki T and Kasuga M: Induction of apoptosis by stomach cancer-associated protein-tyrosine phosphatase-1. J Biol Chem 277: 34359-34366, 2002.

10. Baylin SB and Jones PA: A decade of exploring the cancer epigenome - biological and translational implications. Nat Rev Cancer 11: 726-734, 2011.

11. Heller G, Zielinski CC and Zöchbauer-Müller S: Lung cancer: From single-gene methylation to methylome profiling. Cancer Metastasis Rev 29: 95-107, 2010.

12. Arai E, Chiku S, Mori T, Gotoh M, Nakagawa T, Fujimoto H and Kanai Y: Single-CpG-resolution methylome analysis identifies clinicopathologically aggressive $\mathrm{CpG}$ island methylator phenotype clear cell renal cell carcinomas. Carcinogenesis 33: 1487-1493, 2012.

13. Sato T, Arai E, Kohno T, Tsuta K, Watanabe S, Soejima K, Betsuyaku T and Kanai Y: DNA methylation profiles at precancerous stages associated with recurrence of lung adenocarcinoma. PLoS One 8: e59444, 2013.

14. Sato T, Arai E, Kohno T, Takahashi Y, Miyata S, Tsuta K, Watanabe S, Soejima K, Betsuyaku T and Kanai Y: Epigenetic clustering of lung adenocarcinomas based on DNA methylation profiles in adjacent lung tissue: Its correlation with smoking history and chronic obstructive pulmonary disease. Int J Cancer 135: 319-334, 2014.

15. Jurinke C, Denissenko MF, Oeth P, Ehrich M, van den Boom D and Cantor CR: A single nucleotide polymorphism based approach for the identification and characterization of gene expression modulation using MassARRAY. Mutat Res 573: 83-95, 2005.

16. Fan J, Upadhye S and Worster A: Understanding receiver operating characteristic (ROC) curves. CJEM 8: 19-20, 2006.

17. Seo $\mathrm{Y}$, Matozaki T, Tsuda M, Hayashi $\mathrm{Y}$, Itoh $\mathrm{H}$ and Kasuga M: Overexpression of SAP-1, a transmembrane-type protein tyrosine phosphatase, in human colorectal cancers. Biochem Biophys Res Commun 231: 705-711, 1997.

18. Motiwala T, Majumder S, Kutay H, Smith DS, Neuberg DS, Lucas DM, Byrd JC, Grever M and Jacob ST: Methylation and silencing of protein tyrosine phosphatase receptor type $\mathrm{O}$ in chronic lymphocytic leukemia. Clin Cancer Res 13: 3174-3181, 2007.

19. Motiwala T, Kutay H, Ghoshal K, Bai S, Seimiya H, Tsuruo T, Suster S, Morrison C and Jacob ST: Protein tyrosine phosphatase receptor-type O (PTPRO) exhibits characteristics of a candidate tumor suppressor in human lung cancer. Proc Natl Acad Sci USA 101: 13844-13849, 2004.

20. Veeriah S, Brennan C, Meng S, Singh B, Fagin JA, Solit DB, Paty PB, Rohle D, Vivanco I, Chmielecki J, et al: The tyrosine phosphatase PTPRD is a tumor suppressor that is frequently inactivated and mutated in glioblastoma and other human cancers. Proc Natl Acad Sci USA 106: 9435-9440, 2009.

21. van Doorn R, Zoutman WH, Dijkman R, de Menezes RX, Commandeur S, Mulder AA, van der Velden PA, Vermeer MH, Willemze R, Yan PS, et al: Epigenetic profiling of cutaneous T-cell lymphoma: Promoter hypermethylation of multiple tumor suppressor genes including BCL7a, PTPRG, and p73. J Clin Oncol 23: 3886-3896, 2005.

22. Wang JF and Dai DQ: Metastatic suppressor genes inactivated by aberrant methylation in gastric cancer. World J Gastroenterol 13: 5692-5698, 2007.

23. Oka T, Ouchida M, Koyama M, Ogama Y, Takada S, Nakatani Y, Tanaka T, Yoshino T, Hayashi K, Ohara N, et al: Gene silencing of the tyrosine phosphatase SHP1 gene by aberrant methylation in leukemias/lymphomas. Cancer Res 62: 63906394, 2002.

24. Koyama M, Oka T, Ouchida M, Nakatani Y, Nishiuchi R, Yoshino T, Hayashi K, Akagi T and Seino Y: Activated proliferation of B-cell lymphomas/leukemias with the SHP1 gene silencing by aberrant $\mathrm{CpG}$ methylation. Lab Invest 83: 1849-1858, 2003 . 
25. Reddy J, Shivapurkar N, Takahashi T, Parikh G, Stastny V, Echebiri C, Crumrine K, Zöchbauer-Müller S, Drach J, Zheng Y, et al: Differential methylation of genes that regulate cytokine signaling in lymphoid and hematopoietic tumors. Oncogene 24 732-736, 2005

26. Yeh SH, Wu DC, Tsai CY, Kuo TJ, Yu WC, Chang YS, Chen CL, Chang CF, Chen DS and Chen PJ: Genetic characterization of fas-associated phosphatase-1 as a putative tumor suppressor gene on chromosome $4 \mathrm{q} 21.3$ in hepatocellular carcinoma. Clin Cancer Res 12: 1097-1108, 2006.
27. Jacob ST and Motiwala T: Epigenetic regulation of protein tyrosine phosphatases: Potential molecular targets for cancer therapy. Cancer Gene Ther 12: 665-672, 2005.

28. Wälchli S, Espanel $X$ and Hooft van Huijsduijnen R: Sap-1/PTPRH activity is regulated by reversible dimerization. Biochem Biophys Res Commun 331: 497-502, 2005.

29. Sakuraba J, Shintani T, Tani S and Noda M: Substrate specificity of R3 receptor-like protein-tyrosine phosphatase subfamily toward receptor protein-tyrosine kinases. J Biol Chem 288: 23421-23431, 2013. 